\title{
Casimir energy and force induced by an impenetrable flux tube of finite radius
}

\author{
Volodymyr M. Gorkavenko * \\ Department of Physics, Taras Shevchenko National University of Kyiv, \\ 64/13 Volodymyrska str., Kyiv 01601, Ukraine \\ Yurii A. Sitenko ${ }^{\dagger}$ Olexander B. Stepanov \\ Bogolyubov Institute for Theoretical Physics, National Academy of Sciences of Ukraine, \\ 14-b Metrologichna str., Kyiv 03680, Ukraine
}

\begin{abstract}
A perfectly reflecting (Dirichlet) boundary condition at the edge of an impenetrable magnetic-flux-carrying tube of nonzero transverse size is imposed on the charged massive scalar matter field which is quantized outside the tube. We show that the vacuum polarization effects outside the tube give rise to a macroscopic force acting at the increase of the tube radius (if the magnetic flux is held steady). The Casimir energy and force are periodic in the value of the magnetic flux, being independent of the coupling to the space-time curvature scalar. We conclude that a topological defect of the vortex type can polarize the vacuum of only those quantum fields that have masses which are much less than a scale of the spontaneous symmetry breaking.
\end{abstract}

Keywords: vacuum polarization; Casimir effect; magnetic vortex.

PACS numbers: 04.60.Kz, 11.10.Kk, 11.15.Tk, 11.27.+d

\section{Introduction}

Polarization of the vacuum of quantum matter fields under the influence of boundary conditions was studied intensively over more than six decades since Casimir [1] predicted a force between grounded metal plates: the prediction was that the induced vacuum energy in bounded spaces gave rise to a macroscopic force between bounding surfaces, see reviews in Refs. [2] and [3]. The Casimir force between grounded metal plates has now been measured quite accurately and agrees with the theoretical predictions, see, e.g. Refs. [4] and [5], as well as other publications cited in Refs. [2] and [3].

\footnotetext{
*E-mail: gorka@univ.kiev.ua

†E-mail: yusitenko@bitp.kiev.ua

$\ddagger$ E-mail: _pnd_@ukr.net
} 
In the present paper we consider the vacuum energy which is induced by boundary conditions in space that is not bounded but, instead, is not simply connected, being an exterior to a straight infinitely long tube. This setup is inspired by the famous AharonovBohm effect [6], and we are interested in polarization of the vacuum which is due to imposing a boundary condition at the edge of the tube carrying magnetic flux lines inside itself; this may be denoted as the Casimir-Aharonov-Bohm effect (see also [7]).

The vacuum polarization effects which are due to imposing boundary conditions of various types at the cylindrical surfaces were extensively discussed in the literature, see [8] - [13]. In general, the Casimir effect in the presence of a single smooth object (cylinder or sphere) is rather different from that in the presence of two disjoint ones (e.g., plates): new divergences appear, and to tame them one has to sum contributions of quantized matter from both sides of the boundary surface, still this does not help to get rid completely of divergences, see [3] and references therein. In view of this, the conventional prescription which is to subtract vacuum energy of empty Minkowski space-time becomes insufficient for obtaining the meaningful results. Some authors [14, 15] assert that there is no Casimir effect at all in this case. Our concern will not be in the case of an empty tube but, instead, in the case of a tube filled with the magnetic flux lines. We shall follow the author of ref.[16] who proposes to define the Casimir energy for physical systems divided into classes: the difference in vacuum energy of any two systems within the same class should be finite, then the finite Casimir energy has the universal interpretation as a vacuum energy with respect to the vacuum energy of a certain reference system which is common for the whole class. We define a class of physical systems corresponding to the charged matter field which is quantized outside an impenetrable tube with the magnetic flux taking different values; the case of zero flux can be chosen as the reference system. As we shall show, the Casimir energy for this class is unambiguous and finite.

A magnetic flux tube is formed inside a long current-carrying solenoid or simply a magnetized whisker made of a ferromagnetic material, and its effect on the outside vacuum can be studied in laboratory. Otherwise, a flux tube can be formed as a topological defect of the vortex type, appearing after a phase transition with spontaneous breakdown of the gauge symmetry [17, 18]: the condition of its appearance is that the first homotopy group of the group space of the broken symmetry group be nontrivial. The vortex is characterized by flux $2 \pi \hbar c e_{H}^{-1}$, where $e_{H}$ is the coupling constant of the Higgs scalar field to the vortex-forming gauge field; the transverse size of the vortex is of the order of correlation length $\hbar\left(m_{H} c\right)^{-1}$, where $m_{H}$ is the mass of the Higgs scalar field. The issue of vortices is widely discussed in condensed matter physics (e.g. Abrikosov vortices in superconductors, see [19]), as well as in astrophysics and cosmology (e.g. cosmic strings, see [20, 21]). While considering the effect of the vortices on the vacuum of the surrounding quantum matter, the following two circumstances should be kept in mind: 1) the phase with broken symmetry exists outside the vortex which is topological defect, and the vacuum is to be defined only where the phase exists, hence the quantum matter field does not penetrate inside the vortex, obeying a boundary condition at its edge, 2) the coupling constant $(e)$ of the quantum matter field to the vortex-forming gauge field differs, in general, from $e_{H}$ (e.g. $e=e_{H} / 2$ for normal excitations in superconductors).

A simplifying assumption consists in a neglect of a transverse size of the flux tube, i.e. in a use of an approximation of an infinitely thin singular thread. Energy density and other components of the energy-momentum tensor, which are induced in the background of a singular magnetic thread, were studied in [22] (see also [7, 23]). The quantum matter field 
obeys the regularity condition at the location of the thread, and the vacuum polarization effects are periodic in the value of the magnetic flux with the period equal to the London flux quantum $\left(2 \pi \hbar c e^{-1}\right)$; the absolute value of the induced vacuum energy density is maximal at half of the London flux quantum. A shortcoming of the approximation of a singular thread is the power divergence of the induced vacuum energy-momentum tensor in the vicinity of the thread, and, as a consequence, neither Casimir energy (i.e. the induced vacuum energy per unit length of the thread) nor Casimir force can be defined in this approximation.

The transverse size of the magnetic flux tube was taken into account in [24 - 28], where it was shown that the induced vacuum energy per unit length of the tube depends on the configuration of the magnetic field inside the tube, being quadratic in the flux for sufficiently smooth configurations. However, these authors were concerned with the case when the region of the flux was penetrable for the quantum matter field; therefore, their results have no relation to the Casimir-Aharonov-Bohm effect. When the quantum matter field is excluded from the region of the flux (that is appropriate for the interpretation of the flux tube as a topological defect), then the vacuum polarization effects become independent of the details of the magnetic field configuration and depend periodically on the whole flux [29] - [31]; meanwhile the contribution to both the Casimir energy and force which is due to the magnetic flux in the excluded region is well-defined. In the following quantum matter will be represented by the charged massive scalar field. As we shall see, the vacuum energy which is induced outside the flux tube gives rise to a macroscopic force acting at the increase of the tube radius, if the magnetic flux is held steady. Although the induced vacuum energy density depends on the coupling of the scalar field to the space-time curvature scalar, the Casimir energy and force will be shown to be independent of this coupling.

In the next section we define the renormalized induced vacuum energy density in the background of an impenetrable flux tube and review briefly the obtained earlier results as to its behavior in a plane, i.e. when the spatial dimension along the tube is ignored. The Casimir energy and force in a plane are considered in Section 3. The longitudinal dimensions are added in Section 4 where we find the Casimir energy and force in the most general case of a $(d-2)$-tube in $d$-dimensional space. The obtained results are summarized and discussed in Section 5.

\section{Vacuum energy density}

The temporal component of the energy-momentum tensor for the quantized charged scalar field $\Psi(x)$ in flat space-time is given by expression

$$
T_{00}(x)=\frac{1}{2}\left[\partial_{0} \Psi^{\dagger}, \partial_{0} \Psi\right]_{+}-\frac{1}{4}\left[\partial_{0}^{2} \Psi^{\dagger}, \Psi\right]_{+}-\frac{1}{4}\left[\Psi^{\dagger}, \partial_{0}^{2} \Psi\right]_{+}-\left(\xi-\frac{1}{4}\right) \nabla^{2}\left[\Psi^{\dagger}, \Psi\right]_{+},
$$

where $\boldsymbol{\nabla}$ is the covariant spatial derivative involving both affine and bundle connections and the field operator in the case of a static (ultrastatic) background takes form

$$
\Psi\left(x^{0}, \mathbf{x}\right)=\sum_{\lambda} \frac{1}{\sqrt{2 E_{\lambda}}}\left[e^{-\mathrm{i} E_{\lambda} x^{0}} \psi_{\lambda}(\mathbf{x}) a_{\lambda}+e^{\mathrm{i} E_{\lambda} x^{0}} \psi_{-\lambda}(\mathbf{x}) b_{\lambda}^{\dagger}\right]
$$


units $\hbar=c=1$ are used, $a_{\lambda}^{\dagger}$ and $a_{\lambda}\left(b_{\lambda}^{\dagger}\right.$ and $\left.b_{\lambda}\right)$ are the scalar particle (antiparticle) creation and destruction operators satisfying commutation relations; wave functions $\psi_{\lambda}(\mathbf{x})$ form a complete set of solutions to the stationary Klein-Gordon equation

$$
\left(-\nabla^{2}+m^{2}\right) \psi_{\lambda}(\mathbf{x})=E_{\lambda}^{2} \psi(\mathbf{x})
$$

$m$ is the mass of the scalar particle; $\lambda$ is the set of parameters (quantum numbers) specifying the state; $E_{\lambda}=E_{-\lambda}>0$ is the energy of the state; symbol $\sum_{\lambda}$ denotes summation over discrete and integration (with a certain measure) over continuous values of $\lambda$.

As is known for a long time [32, 33, 34, the energy-momentum tensor depends on parameter $\xi$ which couples $\Psi$ to the scalar curvature of space-time even in the case of the vanishing curvature, see (11); conformal invariance is achieved in the limit of vanishing mass $(m=0)$ at $\xi=(d-1)(4 d)^{-1}$, where $d$ is the spatial dimension. Consequently, the density of the induced vacuum energy which is given formally by expression

$$
\varepsilon=\left\langle\operatorname{vac}\left|T_{00}(x)\right| \operatorname{vac}\right\rangle=\sum_{\lambda} E_{\lambda} \psi_{\lambda}^{*}(\mathbf{x}) \psi_{\lambda}(\mathbf{x})-(\xi-1 / 4) \nabla^{2} \bigvee_{\lambda} E_{\lambda}^{-1} \psi_{\lambda}^{*}(\mathbf{x}) \psi_{\lambda}(\mathbf{x})
$$

depends on $\xi$ as well. This poses a question: whether physically measurable effects (e.g. the Casimir force) can be dependent on $\xi$ ?

In the present paper we are considering a static background in the form of the cylindrically symmetric magnetic flux tube of finite transverse size, hence the covariant derivative is $\boldsymbol{\nabla}=\boldsymbol{\partial}-\mathrm{i} e \mathbf{V}$ with the vector potential possessing only one nonvanishing component given by

$$
V^{\varphi}=\Phi / 2 \pi
$$

outside the tube; here $\Phi$ is the value of the magnetic flux and $\varphi$ is the angle in polar $(r, \varphi)$ coordinates on a plane which is transverse to the tube.

The vacuum polarization depends on the choice of a boundary condition at the edge of the tube $\left(r=r_{0}\right)$. We impose, as in [29] - [31], the Dirichlet boundary condition:

$$
\left.\psi_{\lambda}\right|_{r=r_{0}}=0
$$

i.e. quantum matter is assumed to be perfectly reflected from the thence impenetrable flux tube. Other possible choices of a boundary condition will be considered elsewhere.

The solution to (3) outside the magnetic flux tube can be obtained in terms of the cylindrical functions. The formal expression (4) for the vacuum energy density has to be renormalized by subtracting the contribution corresponding to the zero flux. The tube in 3-dimensional space can be obviously generalized to the $(d-2)$-tube in $d$-dimensional space by adding extra $d-3$ dimensions as longitudinal ones. Thus we obtain (for details see [29, 30]):

$$
\begin{aligned}
\varepsilon_{r e n}=(2 \pi)^{1-d} \int d^{d-2} \mathbf{k}_{z} \int_{0}^{\infty} d k k\left(\sqrt{\mathbf{k}_{z}^{2}+k^{2}+m^{2}}\right. & \left.-\frac{\xi-1 / 4}{\sqrt{\mathbf{k}_{z}^{2}+k^{2}+m^{2}}} \triangle\right) \times \\
& \times\left[S\left(k r, k r_{0}\right)-\left.S\left(k r, k r_{0}\right)\right|_{\Phi=0}\right]
\end{aligned}
$$


where

$$
S\left(k r, k r_{0}\right)=\sum_{n \in \mathbb{Z}} \frac{\left[Y_{|n-e \Phi / 2 \pi|}\left(k r_{0}\right) J_{|n-e \Phi / 2 \pi|}(k r)-J_{|n-e \Phi / 2 \pi|}\left(k r_{0}\right) Y_{|n-e \Phi / 2 \pi|}(k r)\right]^{2}}{Y_{|n-e \Phi / 2 \pi|}^{2}\left(k r_{0}\right)+J_{|n-e \Phi / 2 \pi|}^{2}\left(k r_{0}\right)},
$$

$\mathbb{Z}$ is the set of integer numbers, $J_{\mu}(u)$ and $Y_{\mu}(u)$ are the Bessel and the Neumann functions of order $\mu$, the integration over the components of the $(d-2)$-dimensional momentum $\mathbf{k}_{z}$ ranges from $-\infty$ to $\infty$, and $\triangle=\partial_{r}^{2}+r^{-1} \partial_{r}$ is the radial part of the Laplacian operator on the plane which is orthogonal to the $(d-2)$-tube.

Owing to the infinite range of summation, the last expression is periodic in flux $\Phi$ with a period equal to $2 \pi e^{-1}$, i.e. the London flux quantum (in units $c=\hbar=1$ ). Our further analysis concerns the case of $\Phi=(2 n+1) \pi e^{-1}$ when each of the integrals in (7) is the most distinct from zero. Introducing function

$$
G\left(k r, k r_{0}\right)=\left.S\left(k r, k r_{0}\right)\right|_{\Phi=\pi e^{-1}}-\left.S\left(k r, k r_{0}\right)\right|_{\Phi=0},
$$

we rewrite (7) in the case of $d=2$ in the dimensionless form

$$
r^{3} \varepsilon_{r e n}=\alpha_{+}\left(m r_{0}, m r\right)-(\xi-1 / 4) r^{3} \triangle \frac{\alpha_{-}\left(m r_{0}, m r\right)}{r},
$$

where

$$
\alpha_{ \pm}\left(m r_{0}, m r\right)=\frac{1}{2 \pi} \int_{0}^{\infty} d z z\left[z^{2}+\left(\frac{m r_{0}}{\lambda}\right)^{2}\right]^{ \pm 1 / 2} G(z, \lambda z),
$$

and $\lambda=r_{0} / r(\lambda \in[0,1])$.

Functions $\alpha_{+}$and $\alpha_{-}$were numerically calculated at a set of different distances from the axis of the tube in [30, 31] where it was shown that the results can be approximated by the interpolation function in the form

$$
\alpha_{ \pm}\left(x_{0}, x\right)=\left[ \pm e^{-2 x} x^{1 \mp 1 / 2}\right]\left[\left(\frac{x-x_{0}}{x}\right)^{2} \frac{P_{3}^{ \pm}\left(x-x_{0}\right)}{x^{3}}\right] \frac{Q_{3}^{ \pm}\left(x^{2}\right)}{R_{3}^{ \pm}\left(x^{2}\right)}, \quad x>x_{0},
$$

where $x=m r, x_{0}=m r_{0}$ and $P_{n}^{ \pm}(y), Q_{n}^{ \pm}(y), R_{n}^{ \pm}(y)$ are polynomials in $y$ of the $n$-th order with the $x_{0}$-dependent coefficients. First factor in square bracket in (12) describes the large distance behavior in the case of the zero-radius tube (singular thread), second factor in square bracket is an asymptotics at small distances from the edge of the tube, and the last quotient is the intermediate part. Since the flux tube is impenetrable, the $\alpha_{ \pm}$functions vanish at $x \leq x_{0}$.

For the $\alpha_{+}$function we estimate the relative error of the obtained result as $0.1 \%$. It should be noted that nearly $95 \%$ of the integral value is obtained by direct calculation and only nearly $5 \%$ is the contribution from the interpolation. The integration in the case of the $\alpha_{-}$function is performed more quickly and with a higher accuracy, as compared to the case of the $\alpha_{+}$function, because the former tends to zero more rapidly at large distances. In this case the contribution from the interpolation can be estimated as $10^{-3 \%}$ from the total value.

We define function [30]

$$
\tilde{\alpha}_{-}\left(x_{0}, x\right)=r^{3} \triangle \frac{\alpha_{-}\left(x_{0}, x\right)}{r}=\alpha_{-}\left(x_{0}, x\right)-x \frac{\partial \alpha_{-}\left(x_{0}, x\right)}{\partial x}+x^{2} \frac{\partial^{2} \alpha_{-}\left(x_{0}, x\right)}{\partial x^{2}}
$$


and construct the dimensionless vacuum energy density at different values of the coupling to the space-time curvature scalar $(\xi)$ in the form:

$$
r^{3} \varepsilon_{r e n}=\alpha_{+}\left(x_{0}, x\right)-(\xi-1 / 4) \tilde{\alpha}_{-}\left(x_{0}, x\right) .
$$

The behavior of $\alpha_{ \pm}$and $\tilde{\alpha}_{-}$and $r^{3} \varepsilon_{\text {ren }}$ as functions of the distance from the axis of the tube for different values of $r_{0}$ and $\xi$ was analyzed in [29, 30]. Of primary interest is the behavior at the decrease of the tube radius. It seems plausible that this case becomes more similar to the case of the tube of zero radius (singular thread). However there are some peculiarities in the behavior in the vicinity of the tube, and we discuss them following [31]. Let us first recall the exact expressions corresponding to the case of the singular magnetic thread (see [22]):

$$
\begin{aligned}
& \alpha_{+}(0, x)=\frac{x^{3}}{3 \pi^{2}}\left\{\frac{\pi}{2}-2 x K_{0}(2 x)-K_{1}(2 x)+\frac{K_{2}(2 x)}{2 x}-\right. \\
& \left.-\pi x\left[K_{0}(2 x) L_{1}(2 x)+K_{1}(2 x) L_{0}(2 x)\right]\right\}, \\
& \alpha_{-}(0, x)=\frac{x}{\pi^{2}}\left\{\frac{\pi}{2}-2 x K_{0}(2 x)-K_{1}(2 x)-\pi x\left[K_{0}(2 x) L_{1}(2 x)+K_{1}(2 x) L_{0}(2 x)\right]\right\}, \\
& \tilde{\alpha}_{-}(0, x)=-\frac{x}{\pi^{2}}\left[2 x K_{0}(2 x)+K_{1}(2 x)\right] \text {, }
\end{aligned}
$$

where $K_{\nu}(u)$ and $L_{\nu}(u)$ are the Macdonald and the modified Struve functions of order $\nu$. Consequently, in the vicinity of a thread one gets

$$
\begin{aligned}
& \alpha_{+}(0, x)=\frac{1-3 x^{2}}{12 \pi^{2}}, \quad x \ll 1 \\
& \alpha_{-}(0, x)=-\frac{1-\pi x+(3-2 \gamma-2 \ln x) x^{2}}{2 \pi^{2}}+O\left(x^{3}\right), \quad x \ll 1, \\
& \tilde{\alpha}_{-}(0, x)=-\frac{1}{2 \pi^{2}}+\frac{1+2 \gamma+2 \ln x}{2 \pi^{2}} x^{2}+O\left(x^{3}\right), \quad x \ll 1,
\end{aligned}
$$

where $\gamma$ is the Euler constant. Using the latter relations, we get asymptotics of the renormalized vacuum energy density at small distances from the singular magnetic thread

$$
r^{3} \varepsilon_{r e n}^{\text {sing }}=\frac{1}{12 \pi^{2}}-\frac{x^{2}}{4 \pi^{2}}-\left(\xi-\frac{1}{4}\right)\left(-\frac{1}{2 \pi^{2}}+\frac{1+2 \gamma+2 \ln x}{2 \pi^{2}} x^{2}\right)+O\left(x^{3}\right), \quad x \ll 1 .
$$

In contrast to (18) and (19), the $\alpha_{ \pm}\left(x_{0}, x\right)$ functions in the case of nonzero radius are vanishing quadratically in the vicinity of the tube, see [30],

$$
\left.\alpha_{ \pm}\left(x_{0}, x\right)\right|_{x \rightarrow x_{0}} \sim O\left[\left(x-x_{0}\right)^{2}\right] .
$$

To be more precise, we assume the asymptotics in the form, cf. (12),

$$
\alpha_{ \pm}\left(x_{0}, x\right)= \pm \frac{\left(x-x_{0}\right)^{2}}{x^{2}} f_{ \pm}\left(x_{0}, x\right)
$$

then one gets

$$
\begin{aligned}
\tilde{\alpha}_{-}\left(x_{0}, x\right)=-\left(x-x_{0}\right)^{2} \frac{\partial^{2}}{\partial x^{2}} f_{-}\left(x_{0}, x\right)+\left(1-6 \frac{x_{0}}{x}+5 \frac{x_{0}^{2}}{x^{2}}\right) x \frac{\partial}{\partial x} f_{-}\left(x_{0}, x\right)- \\
\left.-\left(1-8 \frac{x_{0}}{x}+9 \frac{x_{0}^{2}}{x^{2}}\right) f_{(} x_{0}, x\right)
\end{aligned}
$$



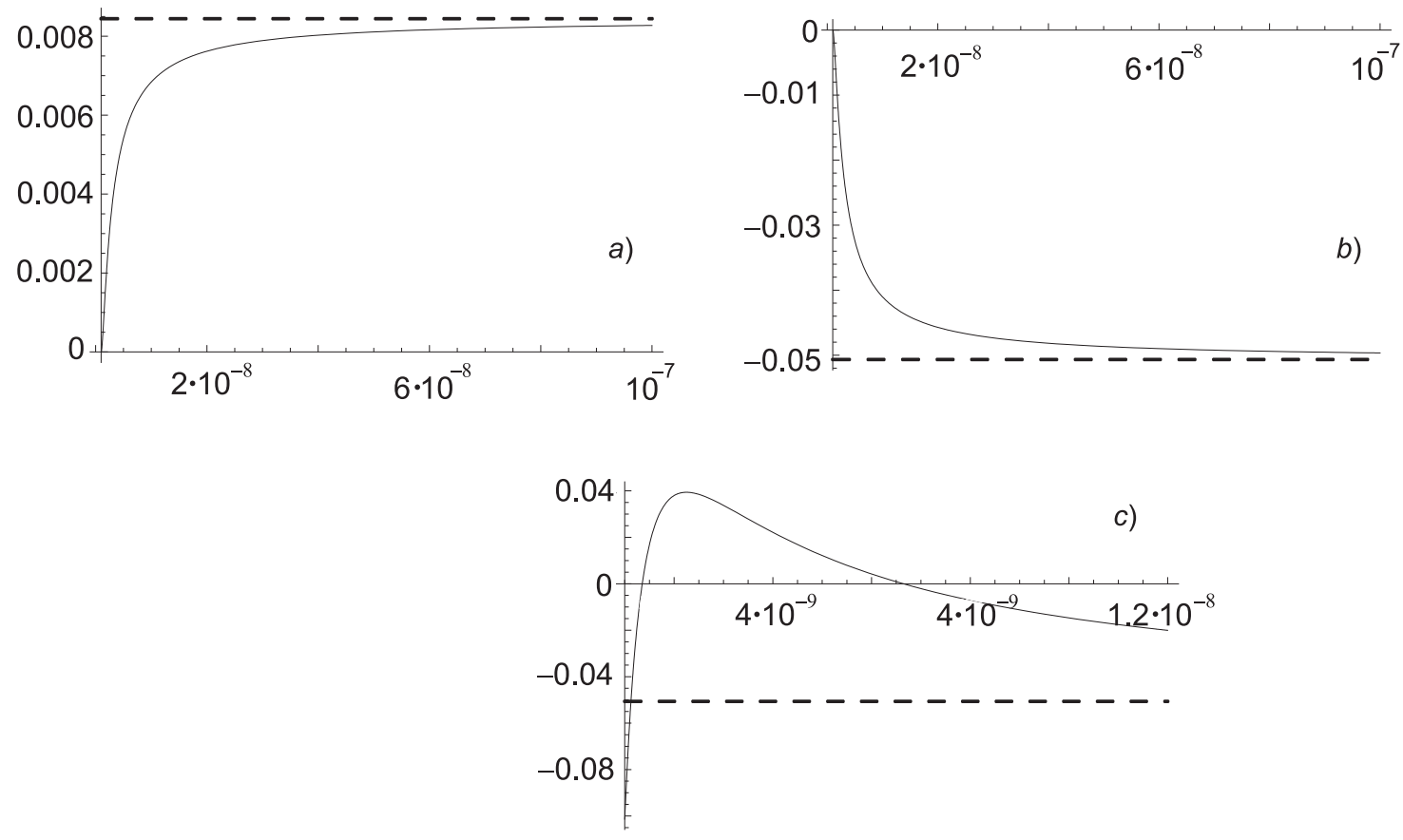

Figure 1: The behavior of the constituents of the dimensionless vacuum energy density at small distances from the tube: a) $\left.\left.\alpha_{+}, b\right) \alpha_{-}, c\right) \tilde{\alpha}_{-}$for the case of $x_{0}=10^{-9}$ (solid line). The behavior of the corresponding functions for the case of a singular magnetic thread is presented by a dashed line. Variable $x\left(x>x_{0}\right)$ is along the abscissa axis.

with $\tilde{\alpha}_{-}\left(x_{0}, x_{0}\right)=-2 f_{-}\left(x_{0}, x_{0}\right)$.

The $f_{ \pm}\left(x_{0}, x\right)$ functions are adjusted as

$$
\begin{aligned}
& f_{+}(0, x)=\frac{1-3 x^{2}}{12 \pi^{2}}, \quad x \ll 1, \\
& f_{-}(0, x)=\frac{1-\pi x+(3-2 \gamma-2 \ln x) x^{2}}{2 \pi^{2}}, \quad x \ll 1 ;
\end{aligned}
$$

consequently, one gets

$$
\begin{aligned}
\left.\tilde{\alpha}_{-}\left(x_{0}, x\right)\right|_{\substack{x_{0} \rightarrow 0 \\
x \rightarrow x_{0}}}=-\frac{1}{2 \pi^{2}}+\frac{1+2 \gamma+2 \ln x}{2 \pi^{2}} & x^{2}+\frac{4-\pi x}{\pi^{2} x} x_{0}+ \\
& +\frac{-9+4 \pi x-7 x^{2}+2 \gamma x^{2}+2 x^{2} \ln x}{2 \pi^{2} x^{2}} x_{0}^{2} .
\end{aligned}
$$

The asymptotical behavior of the $\alpha_{ \pm}$and $\tilde{\alpha}_{-}$functions with the use of (23) - (27) is presented on Fig. 1 for the case of a sufficiently small value of $x_{0}$. It should be noted that the $f_{ \pm}\left(x_{0}, x\right)$ functions depend strongly on $x_{0}$. 


\section{Total vacuum energy and the Casimir force in a plane}

The total vacuum energy which is induced in a plane outside the magnetic flux region is

$$
E_{2}=2 \pi m\left[\int_{x_{0}}^{\infty} \frac{\alpha_{+}\left(x_{0}, x\right)}{x^{2}} d x-\left(\xi-\frac{1}{4}\right) \int_{x_{0}}^{\infty} \frac{\tilde{\alpha}_{-}\left(x_{0}, x\right)}{x^{2}} d x\right]
$$

In view of the relation

$$
\int_{x_{0}}^{\infty} \frac{\tilde{\alpha}_{-}\left(x_{0}, x\right)}{x^{2}} d x=-\left.x \frac{\partial}{\partial x}\left(\frac{\alpha_{-}\left(x_{0}, x\right)}{x}\right)\right|_{x=x_{0}}
$$

which follows from (13), and relations (23) and (26), we conclude that the total vacuum energy is independent of the coupling to the space-time curvature scalar $(\xi)$ :

$$
E_{2}=m \mathcal{D}\left(m r_{0}\right)
$$

where

$$
\mathcal{D}\left(x_{0}\right)=2 \pi \int_{x_{0}}^{\infty} \frac{\alpha_{+}\left(x_{0}, x\right)}{x^{2}} d x .
$$

This is in contrast to the case of the singular magnetic thread, when the total induced vacuum energy is divergent and $\xi$-dependent (see [22]):

$$
E_{2}^{s i n g} \equiv \int_{0}^{2 \pi} d \varphi \int_{0}^{\infty} \varepsilon_{r e n}^{s i n g} r d r \sim 4 m\left(\xi-\frac{1}{12}\right) \int_{0} \frac{d x}{x^{2}}
$$

It is curious that the vacuum energy in this case is finite at $\xi=1 / 12$, being equal to

$$
\begin{aligned}
\left.E_{2}^{\text {sing }}\right|_{\xi=1 / 12}= & \frac{2 m}{3 \pi} \int_{0}^{\infty}\left\{\frac{\pi}{2}-\left(2 x+\frac{1}{2 x}\right) K_{0}(2 x)-K_{1}(2 x)-\right. \\
& \left.-\pi x\left[K_{0}(2 x) L_{1}(2 x)+K_{1}(2 x) L_{0}(2 x)\right]\right\} x d x=-0.01989 \times 2 \pi m
\end{aligned}
$$

and taking the negative value.

Although vacuum energy $E_{2}(30)$ is finite, its value grows infinitely as $x_{0}$ tends to zero (see (23) and (25) ):

$$
\left.E_{2}\right|_{x_{0} \rightarrow 0}=m\left[\frac{1}{18 \pi x_{0}}-\frac{x_{0}}{\pi} \ln x_{0}+O\left(x_{0}^{3}\right)\right],
$$

which is in accordance with the divergence of the vacuum energy in the case of the singular magnetic thread. To be more precise, relation (29) fails to yield zero in the case $x_{0}=0$, and, therefore, the divergence of the vacuum energy in the latter case becomes $\xi$-dependent.

We present the values of vacuum energy $E_{2}(30)$ for several values of the tube radius in the second row of the Table. 

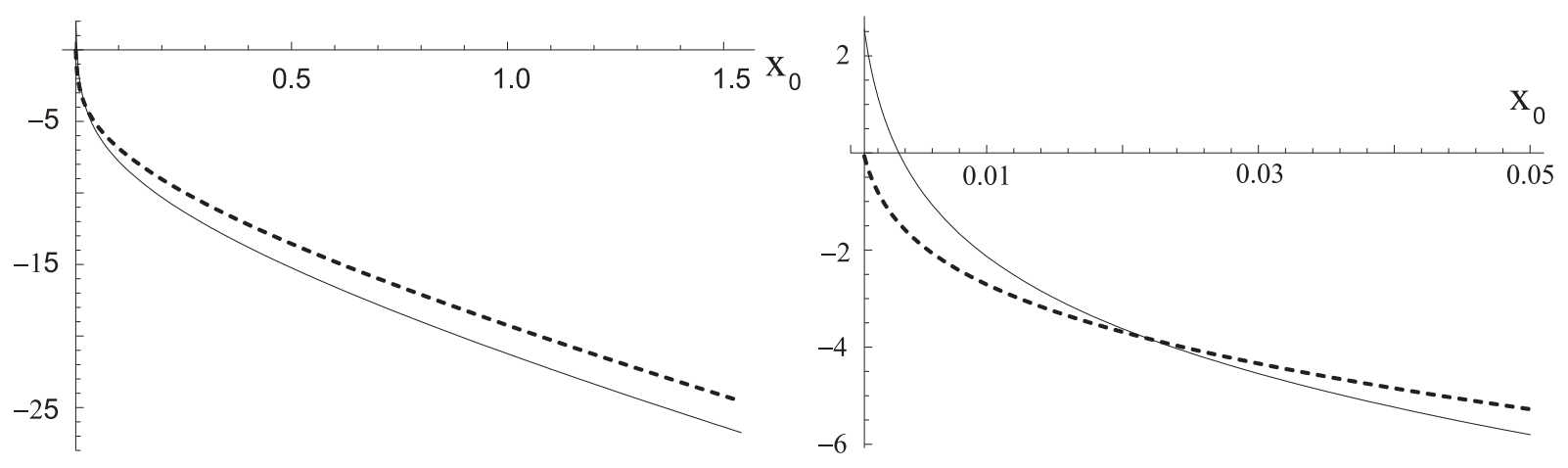

Figure 2: The logarithm of the induced vacuum energy in the plane as a function of the tube radius starting from $x_{0}=10^{-3}: \ln \frac{E_{2}}{m}$ is given by a dashed curve and $\ln \frac{E_{3}}{m^{2}}$ (see Section 4) is given by a solid curve.

\begin{tabular}{|c|c|c|c|c|c|c|}
\hline$m r_{0}$ & $3 / 2$ & 1 & $1 / 2$ & $10^{-1}$ & $10^{-2}$ & $10^{-3}$ \\
\hline$E_{2} / m$ & $3.15 \cdot 10^{-11}$ & $4.363 \cdot 10^{-9}$ & $1.299 \cdot 10^{-6}$ & $1.038 \cdot 10^{-3}$ & 0.0666 & 0.933 \\
\hline$E_{3} / m^{2}$ & $3.577 \cdot 10^{-12}$ & $5.942 \cdot 10^{-10}$ & $2.411 \cdot 10^{-7}$ & $4.162 \cdot 10^{-4}$ & 0.119 & 12.704 \\
\hline
\end{tabular}

Table 1. Values of the dimensionless vacuum energy at several values of $m r_{0}$.

These results are also given on Fig.2 in a logarithmic scale, where the dots corresponding to the data in the Table are joined with the help of an interpolation function $\eta\left(x_{0}\right)=\ln \frac{E_{2}}{m}$, which, for the range $x_{0}>10^{-3}$, can be taken in the form

$$
\eta\left(x_{0}\right)=-a-x_{0}^{b} P_{n}\left(x_{0}\right)-\left(c+x_{0}^{d} Q_{n}\left(x_{0}\right)\right) \ln x_{0} .
$$

where $a, b, c, d$ are the positive constants and $P_{n}\left(x_{0}\right), Q_{n}\left(x_{0}\right)$ are polynomials in $x_{0}$ of the $n$-th order.

To change the radius of the flux tube one has to apply a work that is equal to the change of the total vacuum energy which is induced outside the tube. In the case of the infinitely small change of the radius one has

$$
\delta E_{2}=2 \pi P_{2} r_{0} \delta r_{0}
$$

where $P_{2}$ can be interpreted as the vacuum pressure which acts from the outside to the inside of the tube

$$
P_{2}=\frac{1}{2 \pi r_{0}} \frac{\mathrm{d} E_{2}}{\mathrm{~d} r_{0}}=\frac{m^{3}}{2 \pi x_{0}} \mathcal{D}^{\prime}\left(x_{0}\right)
$$

$\mathcal{D}^{\prime}\left(x_{0}\right)=\frac{\mathrm{d}}{\mathrm{d} x_{0}} \mathcal{D}\left(x_{0}\right)$, and the value of the magnetic flux inside the tube is assumed to remain unchanged.

This results in the Casimir force acting from the inside to the outside of the tube

$$
F_{2}=-2 \pi r_{0} P_{2}=-m^{2} \mathcal{D}^{\prime}\left(x_{0}\right) .
$$

As the tube radius tends to zero, the Casimir force grows infinitely:

$$
F_{2}=m^{2}\left(\frac{1}{18 \pi x_{0}^{2}}-\frac{1}{\pi}\left(\ln x_{0}+1\right)+O\left(x_{0}^{2}\right)\right) \text {. }
$$



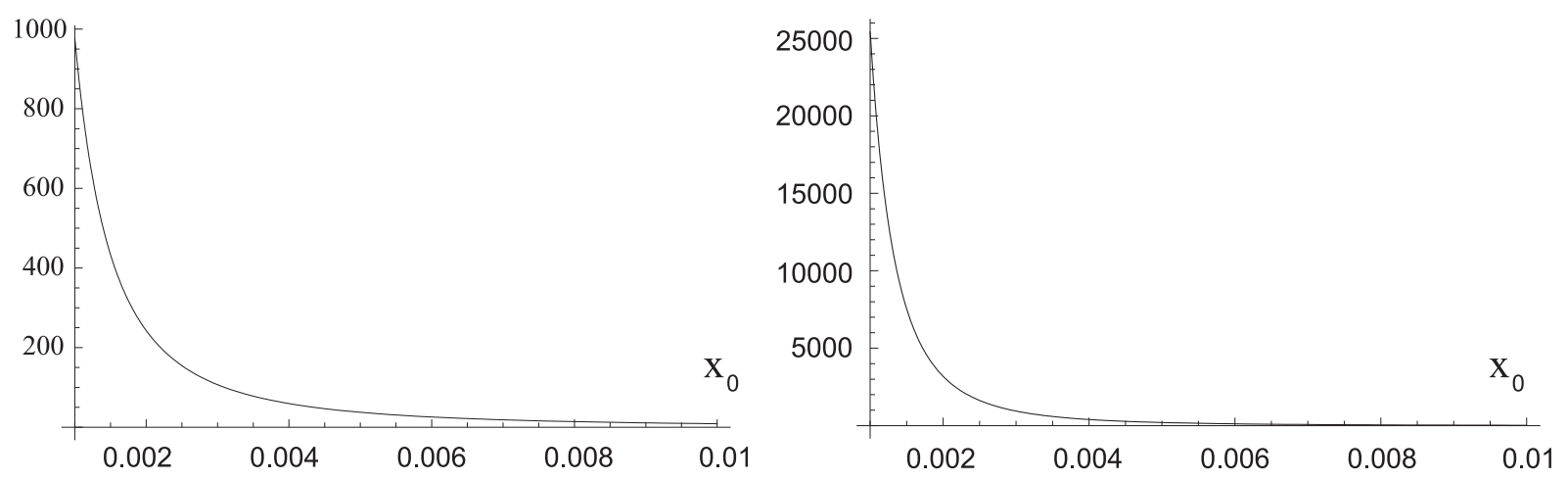

Figure 3: The Casimir force as a function of the tube radius in the range $10^{-3}<x_{0}<10^{-2}$ : $F_{2} / m^{2}$ is on the left and $F_{3} / m^{3}$ (see Section 4) is on the right.

The behavior of the Casimir force is presented on Fig.3.

As one can see, the Casimir force tends to increase the radius of the tube and to minimize the induced vacuum energy of the quantized scalar field. As to the energy stored inside the tube, it is the purely classical energy of the magnetic field. Its behavior at the increase of the tube radius as the magnetic flux is held constant can be different depending on the details of the magnetic field configuration. Mild assumptions as to the smoothness of the configuration yield that the energy is either constant or decreasing at most as $\sim r_{0}^{-2}$.

\section{Generalization to higher than two dimensions}

In $d$-dimensional space, we define the vacuum energy which is induced outside a $(d-2)$ tube in a plane orthogonal to it:

$$
E_{d}=\int_{0}^{2 \pi} d \varphi \int_{r_{0}}^{\infty} d r r \varepsilon_{r e n}
$$

where $\varepsilon_{r e n}$ is given by (7). Generalizing relation (29) we obtain relation

$$
\begin{aligned}
\int_{r_{0}}^{\infty} d r & r \Delta d^{d-2} \mathbf{k}_{z} \int_{0}^{\infty} \frac{d k k}{\sqrt{\mathbf{k}_{z}^{2}+k^{2}+m^{2}}}\left[S\left(k r, k r_{0}\right)-\left.S\left(k r, k r_{0}\right)\right|_{\Phi=0}\right]= \\
& =-\left.\left\{r \partial_{r} \int d^{d-2} \mathbf{k}_{z} \int_{0}^{\infty} \frac{d k k}{\sqrt{\mathbf{k}_{z}^{2}+k^{2}+m^{2}}}\left[S\left(k r, k r_{0}\right)-\left.S\left(k r, k r_{0}\right)\right|_{\Phi=0}\right]\right\}\right|_{r=r_{0}}
\end{aligned}
$$

The right-hand side of (41) is obviously vanishing due to relation

$$
\left.\left[r \partial_{r} S\left(k r, k r_{0}\right)\right]\right|_{r=r_{0}}=0,
$$

stemming from the definition of $S\left(k r, k r_{0}\right)$, see (8) $)$. Consequently, the Casimir energy, i.e. the induced vacuum energy per unit length of the $(d-2)$-tube, is independent of the 
coupling to the space-time curvature scalar

$$
E_{d}=(2 \pi)^{2-d} \int_{r_{0}}^{\infty} d r r \int d^{d-2} \mathbf{k}_{z} \int_{0}^{\infty} d k k \sqrt{\mathbf{k}_{z}^{2}+k^{2}+m^{2}}\left[S\left(k r, k r_{0}\right)-\left.S\left(k r, k r_{0}\right)\right|_{\Phi=0}\right] .
$$

Changing the order of integration over $r$ and $\mathbf{k}_{z}$, we relate $E_{d}$ to the total induced vacuum energy in the $d=2$ case, $E_{2}(\underline{30})$ :

$$
E_{d}=m^{d-1} \frac{(4 \pi)^{1-d / 2}}{\Gamma(d / 2)} \int_{0}^{\infty} d u \sqrt{1+u^{2 /(d-2)}} \mathcal{D}\left(x_{0} \sqrt{1+u^{2 /(d-2)}}\right)
$$

where $u=\left(\left|\mathbf{k}_{z}\right| r_{0}\right)^{d-2}, \Gamma(y)$ is the Euler gamma function, and

$$
\mathcal{D}(y)=\int_{y}^{\infty} \frac{d x}{x^{2}} \int_{0}^{\infty} d z z \sqrt{z^{2}+x^{2}}\left[S\left(z, z \frac{y}{x}\right)-\left.S\left(z, z \frac{y}{x}\right)\right|_{\Phi=0}\right]
$$

is generalizing (31) to arbitrary values of the flux.

Similarly to the $d=2$ case we define the Casimir force acting from the inside to the outside of the $(d-2)$-tube along the radial direction

$$
F_{d}=-\frac{\mathrm{d} E_{d}}{\mathrm{~d} r_{0}}
$$

and relate it to the Casimir force in the $d=2$ case, $F_{2}$ (38):

$$
F_{d}=-m^{d} \frac{(4 \pi)^{1-d / 2}}{\Gamma(d / 2)} \int_{0}^{\infty} d u\left(1+u^{2 /(d-2)}\right) \mathcal{D}^{\prime}\left(x_{0} \sqrt{1+u^{2 /(d-2)}}\right)
$$

It should be emphasized that relations (44) and (47) are valid for arbitrary values of the flux. The finiteness of integrals in (44) and (47) is due to the sufficiently strong decrease of $\mathcal{D}\left(x_{0}\right)$ and $\mathcal{D}^{\prime}\left(x_{0}\right)$ at $x_{0} \gg 1$, which was demonstrated for $\Phi=(2 n+1) \pi e^{-1}$ in the previous section.

Changing the integration variable in (44) and (47), we get

$$
\begin{aligned}
& E_{d}=\frac{2}{r_{0}^{d-1}} \frac{(4 \pi)^{1-d / 2}}{\Gamma\left(\frac{d-2}{2}\right)} \int_{x_{0}}^{\infty} d v v^{2}\left(v^{2}-x_{0}^{2}\right)^{\frac{d-4}{2}} \mathcal{D}(v), \\
& F_{d}=-\frac{2}{r_{0}^{d}} \frac{(4 \pi)^{1-d / 2}}{\Gamma\left(\frac{d-2}{2}\right)} \int_{x_{0}}^{\infty} d v v^{3}\left(v^{2}-x_{0}^{2}\right)^{\frac{d-4}{2}} \mathcal{D}^{\prime}(v),
\end{aligned}
$$

where the latter in the case of $d>3$, after integration by parts, takes form

$$
F_{d}=\frac{2}{r_{0}^{d}} \frac{(4 \pi)^{1-d / 2}}{\Gamma\left(\frac{d-2}{2}\right)} \int_{x_{0}}^{\infty} d v v^{2}\left(v^{2}-x_{0}^{2}\right)^{\frac{d-6}{2}}\left[(d-1) v^{2}-3 x_{0}^{2}\right] \mathcal{D}(v)
$$


At $x_{0} \ll 1$ we obtain

$$
F_{d}=\frac{d-1}{r_{0}} E_{d}=\frac{C_{\Phi}(d)}{r_{0}^{d}}, \quad r_{0} \ll m^{-1},
$$

where

$$
C_{\Phi}(d)=\frac{2(4 \pi)^{1-d / 2}}{\Gamma\left(\frac{d-2}{2}\right)}(d-1) \int_{0}^{\infty} d v v^{d-2} \mathcal{D}(v), \quad d>2,
$$

is monotonically decreasing with the increase of $d$. The numerical estimate of $C(d)$ at $\Phi=(2 n+1) \pi e^{-1}$ in the range $3 \leq d \leq 10$ yields that it can be well approximated as

$$
C_{\Phi}(d)=(d-1) d^{10.025} \exp \left(\frac{44.76}{d}-3 d-28.097\right)
$$

where a decisive factor is $e^{-3 d}$. In the following we shall use a rough, but quite suitable for a further analysis, approximation

$$
C_{\Phi}(d) \approx(d-1) e^{-2.7 d-4}
$$

that is valid for $d \leq 10$. In view of (154) and relation

$$
\frac{1}{d-2} \int_{x_{0}}^{\infty} d v v\left(v^{2}-x_{0}^{2}\right)^{\frac{d-2}{2}}\left[2 \mathcal{D}^{\prime}(v)+v \mathcal{D}^{\prime \prime}(v)\right] \leq(d-1) \int_{0}^{\infty} d v v^{d-2} \mathcal{D}(v),
$$

where the equality sign corresponds to sufficiently small values of $x_{0}$, we find that the dimensionless force, $m^{-d} F_{d}$, as a function of $d$ at $\Phi=(2 n+1) \pi e^{-1}$ can be approximated as

$$
m^{-d} F_{d} \approx(d-1) e^{-4} e^{-d\left(\ln x_{0}+2.7\right)} .
$$

Thus the dimensionless force increases with $d$ at $x_{0} \lesssim e^{-2.7}$ and decreases with $d$ at $x_{0} \gtrsim e^{-2.7}$

In the $d=3$ case we get

$$
E_{3}=\frac{m^{2}}{\pi} \int_{0}^{\infty} d u \sqrt{1+u^{2}} \mathcal{D}\left(x_{0} \sqrt{1+u^{2}}\right)=-\frac{1}{\pi r_{0}^{2}} \int_{x_{0}}^{\infty} d v \sqrt{v^{2}-x_{0}^{2}}\left[\mathcal{D}(v)+v \mathcal{D}^{\prime}(v)\right]
$$

and

$$
F_{3}=-\frac{m^{3}}{\pi} \int_{0}^{\infty} d u\left(1+u^{2}\right) \mathcal{D}^{\prime}\left(x_{0} \sqrt{1+u^{2}}\right)=\frac{1}{\pi r_{0}^{3}} \int_{x_{0}}^{\infty} d v v \sqrt{v^{2}-x_{0}^{2}}\left[2 \mathcal{D}^{\prime}(v)+v \mathcal{D}^{\prime \prime}(v)\right]
$$

We present the values of Casimir energy $E_{3}(157)$ at $\Phi=(2 n+1) \pi e^{-1}(n \in \mathbb{Z})$ for several values of the tube radius in the third row of the Table. These results are also given on Fig. 2 in a logarithmic scale, where the dots corresponding to the data in the Table are joined with help of an interpolation function similarly to that as in the previous section; the comparison is made with the $d=2$ case. Casimir force $F_{3}$ (58) is presented on the 
right of Fig.3 and compared with Casimir force $F_{2}(38)$; the former attains a considerable value of $2.54 \cdot 10^{4} \cdot \mathrm{m}^{3}$ at $r_{0}=10^{-3} \mathrm{~m}^{-1}$.

At $x_{0} \ll 1$, restoring constants $\hbar$ and $c$, we obtain

$$
F_{3}=\frac{2}{r_{0}} E_{3}=\frac{\hbar c}{r_{0}^{3}} C_{\Phi}(3), \quad r_{0} \ll m^{-1},
$$

where, see (52),

$$
C_{\Phi}(3)=\frac{2}{\pi} \int_{0}^{\infty} d v v \mathcal{D}(v) .
$$

Let us compare Casimir force $F_{3}$ (59) with the force caused by the classical magnetic field inside the tube. Assuming the uniformity of the magnetic field filling completely the tube, $B=\Phi /\left(\pi r_{0}^{2}\right)$, one obtains an expression for the classical energy per unit length of the tube, $E^{(\text {class })}=\Phi^{2} /\left(2 \pi r_{0}^{2}\right)$, which can be rewritten in terms of London flux quantum $\Phi_{0}=2 \pi \hbar c e^{-1}$ and fine structure constant $\alpha=e^{2}(4 \pi \hbar c)^{-1}$ :

$$
E^{(\text {class })}=\frac{\hbar c}{r_{0}^{2}} \frac{\Phi^{2}}{\Phi_{0}^{2}} \frac{1}{2 \alpha} .
$$

The classical force which tends to decrease energy (61) by increasing the tube radius under the steady magnetic flux filling completely the tube is

$$
F^{(\text {class })}=-\frac{\mathrm{d}}{\mathrm{d} r_{0}} E^{(\text {class })}=\frac{\hbar c}{r_{0}^{3}} \frac{\Phi^{2}}{\Phi_{0}^{2}} \frac{1}{\alpha} .
$$

Comparing this with Casimir force $F_{3}$ (59), we recall that $C_{\Phi}(3)$ is a periodic function of the magnetic flux, vanishing at $\Phi=n \Phi_{0}$. Even the maximal value of $C_{\Phi}(3)$ which is achieved at $\Phi=(n+1 / 2) \Phi_{0}$ and is equal to $2.545 \cdot 10^{-5}$, see (54) , is more than million times smaller than the value of the corresponding factor, $\Phi^{2} /\left(\Phi_{0}^{2} \alpha\right)$, in (62): taking $\Phi=\Phi_{0} / 2$ one obtains value $(4 \alpha)^{-1} \approx 34.2$ for this factor.

However, as it was already noted, the classical force acting from the inside of the tube depends strongly on the detailed form of the magnetic field configuration: it decreases if the magnetic field is decreasing in the vicinity of the tube edge. For instance, in the case of the magnetic field concentrated wholly inside a tube of smaller radius, the classical force acting to extend the tube of larger radius disappears at all, and only the Casimir force from the outside vacuum is left in this capacity.

\section{Summary}

In the present paper we consider the vacuum polarization effects which are induced in charged scalar matter by a magnetic flux enclosed in an impenetrable finite-radius tube; a perfectly reflecting (Dirichlet) boundary condition is imposed at the edge of the tube. The previous analysis of the induced vacuum energy density in the $d=2$ case [29, 30] was extended down to the values of the tube radius as small as $r_{0}=10^{-3} \hbar(m c)^{-1}$ in 31, where it was shown that contrary to the case of a singular magnetic thread $\left(r_{0}=0\right)$, the vacuum energy density is finite everywhere, but its behavior is very similar to that in the $r_{0}=0$ case, excepting the behavior in the vicinity of the tube, where peculiar oscillations 
appear. The case of $r_{0}<10^{-3} \hbar(m c)^{-1}$ is analyzed indirectly by combining the numerical and analytical estimates, and the difference between the $r_{0}=0$ and $r_{0}=10^{-9} \hbar(m c)^{-1}$ cases is illustrated by Fig.1.

These two circumstances (the finiteness and at the same time the similarity to the case of a singular thread) which are proven in the $d=2$ case have far-reaching consequences that allow us to determine the finite Casimir energy in the case of space of arbitrary dimension, as long as the tube radius is taken into account. We find that the Casimir energy, i.e. the vacuum energy per unit length of the $(d-2)$-tube, is positive and independent of the coupling to the space-time curvature scalar $(\xi)$, notwithstanding the $\xi$-dependence of the vacuum energy density and its lack of positivity. The functional dependence of the Casimir energy on the tube radius for the magnetic flux equal to half of the London flux quantum is numerically estimated for the range $10^{-3} \hbar(m c)^{-1}<r_{0}<1.5 \hbar(m c)^{-1}$, and the results for the $d=2$ and $d=3$ cases are presented in the Table and on Fig.2. The Casimir energy is negligible for $r_{0} \sim \hbar(m c)^{-1}$, being of order $10^{-10} \cdot m^{d-1} c^{d} \hbar^{2-d}$ for $d=2,3$ and even less for larger $d$, but it increases considerably with the decrease of the tube radius.

The Casimir energy gives rise to the Casimir force which is directed from the inside to the outside of the tube along its normal. The force is $\xi$-independent as well as the Casimir energy. The force acts at the increase of the tube radius and the decrease of the Casimir energy, if the magnetic flux is held steady. The behavior of the force as a function of the tube radius in the $d=2$ and $d=3$ cases for the magnetic flux equal to half of the London flux quantum is illustrated by Fig.3. The force takes considerable values at small values of the tube radius and actually disappears otherwise: in the $d=3$ case it is, e.g., $2.54 \cdot 10^{4} \cdot m^{3} c^{4} \hbar^{-2}$ at $r_{0}=10^{-3} \hbar(m c)^{-1}$ and $10^{-2} \cdot m^{3} c^{4} \hbar^{-2}$ at $r_{0}=10^{-1} \hbar(m c)^{-1}$.

It should be noted that we consider the case of the Casimir force caused by a magnetic flux enclosed by a boundary where the Dirichlet boundary condition is imposed. The force is periodic in the flux value with a period equal to the London flux quantum, attaining its maximal value at $\Phi=(n+1 / 2) \Phi_{0}$ and vanishing at $\Phi=n \Phi_{0}(n \in \mathbb{Z})$. A general conclusion which is valid for arbitrary spatial dimension $d \geq 2$ is that the Casimir energy and force at $r_{0} \ll \hbar(m c)^{-1}$, when they take considerable values, are actually the same as they are in the case of the massless scalar field, see (34), (39) and (151); the massive case becomes formally distinct from the massless one at larger values of the tube radius, when the Casimir energy and force take negligible values. The Casimir force and energy increase with $d$ at smaller $r_{0}$, when they are considerable, while decrease with $d$ at larger $r_{0}$, when they are negligible, see (56); even the comparison of numerical calculations for the $d=2$ and $d=3$ cases reveals this fact, see the Table and Fig.2.

Whereas in the case of parallel plates the pure action of the Casimir force to minimize the Casimir energy leads to a collapse, the pure action of the Casimir force to minimize the Casimir energy in the case of a flux tube leads not to a collapse but to an expansion of the tube in the transverse direction. Note that the classical energy of the constant magnetic flux inside the tube is most likely to be constant or decreasing maximally as $r_{0}^{-2}$ with the expansion of the tube radius, see (61). Thus the Casimir force tends to smear both quantum and classical effects of the flux tube. The vacuum polarization is quite negligible at $m c r_{0}>\hbar$, whereas it becomes noticeable at $m c r_{0} \ll \hbar$. If the flux tube is interpreted as a topological defect of the vortex type, then the vacuum polarization in its background is absent when the mass of the Higgs field $\left(m_{H} \sim \hbar\left(r_{0} c\right)^{-1}\right)$ does not exceed the mass of the quantum matter field, $m_{H} \lesssim m$. Vacuum polarization is essential for the quantum matter field with the mass which is much less than the Higgs mass, $m \ll m_{H}$; 
since $\Phi=2 \pi \hbar c e_{H}^{-1}$ for the topological defect case, the effect is maximal when the coupling of the Higgs field to the gauge field is twice the coupling of the quantum matter field to the gauge field, $e_{H}=2 e$ (e.g. the Higgs field describing the Cooper pair in a superconductor). In particular, we can arrive at a conclusion that a cosmic string which has been formed at the grand unification scale polarizes the vacuum of the present-day quantum matter, but it has no effect on the vacuum of matter fields with masses which are comparable to the scale of grand unification.

\section{Acknowledgments}

The work was partially supported by special program "Microscopic and phenomenological models of fundamental physical processes in micro- and macroworld" of the Department of Physics and Astronomy of the National Academy of Sciences of Ukraine and by the ICTP — SEENET-MTP grant PRJ-09 ""Strings and Cosmology". V.M.G. and Yu.A.S. acknowledge the support from the State Agency for Science, Innovations and Informatization of Ukraine under the SFFR-BRRFR grant F54.1/019.

\section{References}

[1] H.B.G. Casimir, Proc. Kon. Ned. Akad. Wetenschap B 51, 793 (1948); Physica 19, 846 (1953).

[2] K.A. Milton, The Casimir Effect: Physical Manifistations of Zero-Point Energy (World Scientific, River Edge, 2001).

[3] M. Bordag, G.L. Klimchitskaya, U. Mohideen and V.M. Mostepanenko, Advances in the Casimir Effect (Oxford University Press, Oxford, 2009).

[4] S.K. Lamoreax, Phys. Rev. Lett. 78, 5 (1997).

[5] G. Bressi, G. Carugno, R. Onofrio and G. Ruoso, Phys. Rev. Lett. 88, 041804 (2002).

[6] Y. Aharonov and D. Bohm, Phys. Rev. 115, 485 (1959).

[7] Yu.A. Sitenko and A.Yu. Babansky, Mod. Phys. Lett. A 13, 379 (1998).

[8] L.L. DeRaad Jr. and K.A. Milton, Ann. Phys. (N.Y.) 136, 229 (1981).

[9] P. Gosdzinsky and A. Romeo, Phys. Lett. B 441, 265 (1998).

[10] N. Graham, R. L. Jaffe, V. Khemani, M. Quandt, M. Scandurra and H. Weigel, Phys. Lett. B 572, 196 (2003).

[11] S.A. Fulling, J. Phys. A: Math. Theor. 36, 6857 (2003).

[12] G. Barton, Phys. Rev. D 73, 065018 (2006).

[13] I. Cavero-Pelaez, K. A. Milton and K. Kirsten, J. Phys. A: Math. Theor. 40, 3607 (2007). 
[14] A. Bulgac, P. Magierski, A. Wirzba, Phys. Rev. D 73, 025007 (2006).

[15] A. Wirzba, J. Phys. A: Math. Theor. 41, 164003 (2008).

[16] M. Schaden, Phys. Rev. A 73, 042102 (2006).

[17] A.A. Abrikosov, Sov. Phys.-JETP 5, 1174 (1957).

[18] H.B. Nielsen and P. Olesen, Nucl. Phys. B 61, 45 (1973).

[19] R.P. Huebener, Magnetic Flux Structure in Superconductors (Springer-Verlag, Berlin, 1979).

[20] A. Vilenkin and E.P.S. Shellard, Cosmic Strings and Other Topological Defects (Cambridge University Press, Cambridge UK, 1994).

[21] M.B. Hindmarsh and T.W.B. Kibble, Rep. Prog. Phys. 58, 477 (1995).

[22] Yu.A. Sitenko and V.M. Gorkavenko, Phys. Rev. D 67, 085015 (2003).

[23] Yu.A. Sitenko and A.Yu. Babansky, Phys. Atom. Nucl. 61, 790 (1998).

[24] G. Dunne and T.M. Hall, Phys. Lett. B 419, 322 (1998).

[25] M. Bordag and K. Kirsten, Phys. Rev. D 60, 105019 (1999).

[26] M.P. Fry, Intern. J. Mod. Phys. A 17, 936 (2002).

[27] K. Langfeld, L. Moyaerts and H. Gies, Nucl. Phys. B 646, 158 (2002).

[28] N. Graham, V. Khemani, M. Quandt, O. Schroeder and H. Weigel, Nucl. Phys. B 707, 233 (2005).

[29] V.M. Gorkavenko, Yu.A. Sitenko and O.B. Stepanov, J. Phys. A: Math. Theor. 43, 175401 (2010).

[30] V.M. Gorkavenko, Yu.A. Sitenko and O.B. Stepanov, Intern. J. Mod. Phys. A 26, 3889 (2011).

[31] V.M. Gorkavenko, Yu.A. Sitenko and O.B. Stepanov, Ukrain. J. Phys. 58, 424 (2013).

[32] R. Penrose, in: Relativity, Groups and Topology, edited by B.S. DeWitt, C. DeWitt (Gordon and Breach, New York, 1964).

[33] N.A. Chernikov and E.A. Tagirov, Ann. Inst. Henri Poincare A 9, 109 (1968).

[34] C.G. Callan, S. Coleman and R. Jackiw, Ann. Phys. (N.Y.) 59, 42 (1970). 